\title{
METODOLOGÍA PARA ANALIZAR LA INTERACCIÓN ENTRE LO CULTURAL, LO SOCIAL Y LO AFECTIVO EN EDUCACIÓN MATEMÁTICA
}

\author{
Pl anas, Núria \\ Departament de Didàctica de la Matemàtica i de les Ciències Experimentals. Universitat Autònoma de Barcelona \\ Nuria.Planas@uab.es
}

\begin{abstract}
Resumen. Últimamente se viene produciendo un creciente énfasis en aspectos socioculturales y afectivos de la educación matemática. Este artículo presenta un enfoque inclusivo a estos aspectos para mejorar la comprensión del problema de la alternancia entre participación y no-participación en el aula de matemáticas. Se describe una metodología cualitativa microetnográfica basada en tres categorías: $a$ ) la cultural, relativa a diferencias en la interpretación de las normas del aula; $b$ ) la social, relativa a valoraciones dadas a las diferentes interpretaciones; y $c$ ) la afectiva, relativa a respuestas emocionales dadas por los alumnos a normas y valoraciones. Para probar la adecuación de un enfoque inclusivo al problema mencionado, se exponen con detalle métodos interpretativos que han sido usados en Planas (2001).
\end{abstract}

Palabras clave. Educación matemática, teorías sociales del aprendizaje, participación, microetnografía, normas, valoraciones, respuestas emocionales.

Summary. Recently, there has been an increasing emphasis on socio-cultural and affective issues in mathematics education research. This paper presents an interrelated approach to these issues in order to better understand the alternance between participation and non-participation in the mathematics classroom. The methodology exposed is micro-ethnographic and based on three main categories: $a$ ) the cultural, related to differences in the interpretation of classroom norms; $b$ ) the social, related to valorizations given to different interpretations; and $c$ ) the affective, related to emotional responses given by the students to both norms and valorizations. Some interpretive methods that have been used in a recent work (Planas, 2001) are shown in detail as a way to prove the adequacy of the interrelated approach.

Keywords. Mathematics education, social theories of learning, participation, micro-ethnography, norms, valorizations, emotional responses.

\section{ANTECEDENCTES Y JUSTIFICACIÓN}

Cada vez son más los estudios que conciben el aprendizaje matemático como una forma de participación social (Abreu y Cline, 2003; Alro y Skovsmose, 2002; Morgan, 2000). Muchos de estos estudios prestan atención a la pluralidad de significados, valoraciones, legitimidades e identidades coexistentes en el aula y, simultáneamente, a la gestión social de esta pluralidad. El aprendiz de matemáticas es, en estos trabajos, alguien cuyos significados personales no siempre coinciden con los legitimados en el aula, que se encuentra sometido a las valoraciones del entorno y que, además, reconstruye continuamente su identidad en función de las prácticas en las cuales participa y aquéllas en las cuales deja de participar.
Hasta no hace mucho ha predominado una visión esencialmente intrapersonal en el estudio de los procesos de aprendizaje matemático. De acuerdo con este enfoque, el aprendizaje matemático resulta difícil debido a que en los procesos de construcción de conocimientos intervienen aspectos de carácter intrapersonal que son verdaderos obstáculos a superar. Pero, junto con los obstáculos cognitivos derivados de las situaciones intrapersonales en los procesos de aprendizaje, existen obstáculos en la comprensión de las formas de participación en el aula que influyen inevitablemente en estos procesos. Recientes investigaciones sobre el aula de matemáticas desde una perspectiva sociocultural y afectiva integrada ponen 
de manifiesto problemas en el aprendizaje matemático surgidos de las dificultades de los alumnos por intentar comprender y aplicar los elementos legitimados de la dinámica general del aula (Boaler y Greeno, 2000; Evans, 2000; Zevenbergen, 2002).

Lerman (2001) señala la gran dificultad por encontrar modelos metodológicos válidos como uno de los motivos del rechazo al estudio de los elementos socioculturales y emocionales involucrados en el aprendizaje matemático. Aún falta mucho para consolidar líneas de investigación que integren lo afectivo, lo cultural y lo social en educación matemática. Desarrollar metodologías en este sentido es crucial porque ha de facilitar una mejor comprensión de los procesos de aprendizaje de todos los alumnos y, en especial, los de aquéllos pertenecientes a grupos minoritarios en situación de riesgo social cuyas distancias a las formas legitimadas de actuación en el aula a menudo suponen un obstáculo en su propia participación.

\section{CUESTIÓN DE INVESTIGACIÓN Y OBJETI- VOS DEL ESTUDIO}

La cuestión central de investigación que caracteriza el estudio es la siguiente: iSe pueden establecer conexiones entre la comprensión que un alumno tiene de las normas del aula de matemáticas y la aparición de obstáculos en su proceso de aprendizaje matemático? En Planas y Civil (2002) se explica la evolución desde el interés inicial centrado en el tema de la no-participación hasta la presente cuestión.

Buscamos las conexiones desde el supuesto que: $a$ ) la diversidad de interpretaciones de las normas provoca dificultades comunicativas en los participantes del aula de matemáticas; $b$ ) los procesos sociales desarrollados en dicha aula condicionan las oportunidades de resolución de estas dificultades; $c$ ) las dificultades comunicativas y las dificultades para resolverlas influyen en los procesos individuales de aprendizaje matemático; y $d$ ) las conexiones entre comprensión de normas y obstáculos en los procesos de aprendizaje matemático deben buscarse teniendo en cuenta los dos tipos de dificultades apuntados. En particular, formulamos la cuestión de investigación en el caso de jóvenes en situación de riesgo al ser los que acumulan mayores índices de fracaso en la matemática escolar y los que parecen presentar una mayor distancia cultural al contexto normativo académico: ¿Cuáles son las conexiones en el caso de alumnos especialmente distantes de la cultura académica y social representada en el aula de matemáticas de secundaria? Por otra parte, la cuestión de investigación enlaza con una reflexión más general sobre las conexiones entre los procesos intrapersonales e interpersonales de aprendizaje en cualquier aula y la mediación del contexto social: ¿En qué sentido los procesos de aprendizaje están influenciados por las características sociales del contexto donde se producen?

De acuerdo con la cuestión de investigación y a la luz del estado actual de los trabajos en esta área de estudio, enunciamos el siguiente objetivo principal: poner de manifiesto obstáculos en el aprendizaje matemático derivados de la complejidad sociocultural del aula de matemáticas. Este objetivo pretende indagar y documentar dos realidades distintas pero inseparables: la complejidad cultural del aula de matemáticas (la diversidad de interpretaciones de las normas) y la complejidad social (la diversidad de valoraciones y legitimidades). Una vez probada la complejidad sociocultural, se estudia su impacto en los procesos individuales de aprendizaje matemático. Se buscan obstáculos en el aprendizaje que parezcan estar relacionados con la experiencia de interpretaciones no compartidas de las normas del aula, con valoraciones negativas no esperadas por el alumno y con escenarios emocionales asociados a la vivencia del contraste de normas y valoraciones.

La fase empírica se organiza por medio de cinco objetivos específicos que operativizan el objetivo principal y articulan cinco grandes temas:

1) Identificar momentos de interrupción en los procesos individuales de aprendizaje matemático -la fragilidad de las trayectorias de participación en el aula de matemáticas.

Para cada caso de interrupción documentado:

2) Identificar divergencias en la interpretación de las normas sociomatemáticas del aula y distancias culturales emergentes entre los participantes -la complejidad cultural del aula de matemáticas.

3) Identificar valoraciones asociadas al alumno y distancias sociales emergentes entre los participantes -la complejidad social.

4) Identificar respuestas emocionales del alumno ante distancias culturales y sociales vividas tras las experiencias de contraste de normas y valoraciones -la diversidad de experiencias de la complejidad sociocultural.

5) Cuando sea posible, elaborar una ruta explicativa para la interrupción e indagar explicaciones en base a distancias culturales, sociales y experiencias que el alumno tiene de ellas -obstáculos en el aprendizaje matemático.

\section{ENFOQUE METODOLÓGICO}

La aproximación metodológica es básicamente de carácter idiográfico, en el sentido de Cohen y otros (1997). Esto significa, entre otras cosas, que el tipo de estudio y los supuestos adoptados parten de una realidad poliédrica y dinámica del aula y sus participantes. Conocer empíricamente la realidad que nos interesa consiste en conocer las interpretaciones que de ella hacen los que la construyen de forma activa. El acceso a la realidad del aula se produce de modo cualitativo, a través de lo que sus participantes dicen, hacen o sugieren y, por tanto, la interpretación de las interpretaciones de los participantes se convierte en el elemento central del análisis. 
Nuestra interpretación inclusiva del fenómeno de fracaso matemático escolar se fundamenta en las teorías sociales del aprendizaje y, más en concreto, se basa en tres elementos que caracterizan la participación del alumno en el aula (Planas, 2002): a) la identidad, construyéndose, reconstruyéndose y «co-construyéndose» a partir de las interacciones sociales que modelan la participación del alumno en el aula de matemáticas (los procesos de resistencia y adecuación de Zevenbergen $(2002) ; b$ ) el significado, creándose y recreándose en las diferentes formas de participación usadas por los alumnos (las normas sociales y sociomatemáticas de Yackel y Cobb (1996); c) el discurso, produciéndose y reproduciéndose por medio de mecanismos que controlan el acceso del alumno a las diferentes formas de participación (las valoraciones y legitimidades de Lerman, 2001). Esta triple aproximación a los hechos del aula de matemáticas da lugar a nuestras tres categorías de análisis:

1) Categoría cultural: normas usadas o sugeridas en el aula durante el período de participación del alumno, diferentes de las esperadas por él.

2) Categoría social: valoraciones negativas recibidas durante este mismo período.

3) Categoría afectiva: respuestas emocionales manifestadas por el alumno, aparentemente como reacción a normas inesperadas y valoraciones negativas.

Al observar las interacciones sociales del aula para conocer fragmentos de la realidad experiencial del alumno, pretendemos integrar el estudio de factores afectivos, cognitivos, culturales y sociales. Para indagar factores intrapersonales a partir del estudio de factores interpersonales, se ha creído conveniente adoptar un método microetnográfico centrado en un estudio de casos. La microetnografía es idónea cuando se considera que las relaciones a estudiar se han originado básicamente en el entorno donde se centra la recogida principal de datos (Clarke, 1998). Algunas de las investigaciones que orientan la fundamentación empírica, inspiran partes centrales del diseño metodológico y justifican el enfoque microetnográfico son de los siguientes autores:

- Wenger (1998), por la caracterización de las identidades individuales de participación y no-participación en un contexto de prácticas y la aportación de comportamientos que expresan el abandono de la implicación.

- Abreu (1993), por la aplicación de una microetnografía en estudios sobre comunidades de prácticas centrados en aspectos relacionados con la variedad de significados de referencia coexistentes.

- Cobb y Yackel (1998), por el uso de un análisis interaccional al estudiar significados de entrada y de salida no siempre coincidentes en las interacciones sociales del aula de matemáticas.

- Lerman (2000), por el planteamiento de interrogantes conceptuales y metodológicos a los trabajos de Cobb y sus colegas, y por la propuesta de variables de estudio que integren el papel de las valoraciones en los microprocesos de interacción social en el aula de matemáticas.

- Wood (1994), por el análisis microcualitativo de modelos de interacción social en el aula a partir de la simplificación progresiva de elementos narrativos y situacionales del discurso que revelen valoraciones e intencionalidades.

\section{DISEÑO EMPÍRICO}

El estudio tuvo lugar en tres aulas de matemáticas de secundaria con alumnos de entre 16 y 18 años. Se observaron un total de 24 alumnos, 6, 7 y 11 en cada aula. La extrema marginación de los tres centros públicos de Barcelona donde se ubican las aulas explica ratios tan bajas. En un estudio previo (Planas, 1999), se concluyó que los alumnos con identidad social de riesgo son más propensos a hacer visibles sus episodios de no-participación en el aula debido a su habitual falta de competencias escolares básicas («jYa tuve bastante!», «jNo me mires, olvídame!», irse del aula dando un portazo, mirar fijamente a través de la ventana durante mucho rato). Es de suponer, pues, que la selección de este tipo de alumnos habrá de facilitar la identificación de interrupciones por medio de mecanismos sencillos tales como la observación. Una vez elegido el perfil de alumnos, se escogieron aulas conducidas por profesores con los cuales ya se había colaborado en otras ocasiones.

Las sesiones de aula donde se localizan los casos de interrupción se observaron y grabaron en audio y vídeo entre septiembre y diciembre de 1999. El procedimiento para la recogida de datos consistió en escuchar y transcribir las sesiones y, a continuación, completar las transcripciones con las notas de campo escritas por la investigadora durante la sesión o inmediatamente tras ella. El escaso número de alumnos permitió la organización en dos grupos de trabajo, por lo que dos cámaras fueron suficientes para recoger con exactitud palabras, gestos $\mathrm{y}$ acciones de todos los participantes. Optamos por un ambiente de resolución de problemas donde el trabajo en grupo fomentara una continua interacción entre alumno y entorno. La investigación en su totalidad generó una vastísima colección de grabaciones y transcripciones. Para cada caso de interrupción, y con el propósito de buscar causas de tipo intrapersonal, sólo analizamos las grabaciones y transcripciones obtenidas en la sesión donde dicha interrupción se había producido.

\section{MÉTODOS DE ANÁLISIS: EJEMPLIFICACIÓN DE UN CASO}

Durante una sesión de clase en una de las aulas observadas, uno de los alumnos, A1, experimentó una interrupción de larga duración en su proceso de aprendizaje matemático. Este apartado desarrolla los pasos seguidos con el propósito de estudiar en detalle factores sociocultura- 
les y afectivos relacionados con el contexto normativo del aula de matemáticas que puedan haber contribuido a provocar la interrupción.

\section{Identificación de la interrupción}

En primer lugar, se construyen dos coordenadas complementarias, la narrativa y la cronológica. La coordenada narrativa recoge momentos del discurso del aula que ponen de relieve un cambio en la participación del alumno, mientras que la cronológica muestra cuánto dura la etapa de no-participación resultante del cambio. Las coordenadas restantes de localización aportan información adicional para contextualizar de un modo general la interrupción. A continuación, se hace un primer análisis intuitivo del caso centrándonos en la respuesta a cuestiones relativas a la situación del alumno en el aula y ante la tarea matemática a lo largo de la sesión: ¿a quién (no) mira?, ¿quién (no) le mira? I ¿a quién (no) habla?, ¿quién (no) le habla? I ¿a quién (no) escucha?, ¿quién (no) le escucha? / ¿qué reacciones emocionales? ¿qué escenarios emocionales? / ¿qué procesos, qué dificultades, qué éxitos y errores? El análisis intuitivo de estas respuestas decide si es razonable pasar a una fase avanzada del estudio. En este caso, hay elementos del entorno interpersonal del alumno que pueden haber contribuido a provocar cambios en su participación.

\section{Coordenada narrativa}

A1 interviene en la discusión conjunta por iniciativa propia, "el 3 va mal porque es impar», "yo pondría justito lo que piden porque, si nos empezamos a animar, luego el pastel no se va a parecer en na[...]»

Reacciona activamente a los comentarios ajenos, «bueno, yo casi que digo lo de poner lo justito porque te va a salir mejor, pero, si ponemos un poquito más o un poquito menos, no va a pasar na, que el pastel está bueno igual.»

Introduce nuevos elementos en la discusión, «tú te vas a la tienda, al colmadero ése, y compras un cacharro de ésos, un molde de esos pa 10 y te vas echando lo que te quepa, lo meneas to, lo que sobra lo tiras, y ya está, tío.»

Sigue con la mirada a los que toman la palabra y acaba frases empezadas, «eso, al limón le sacas la piel pa tener más pedazos y te salga to más fino.»

Responde groseramente al profesor, «mira, tío, yo no sé hacer pasteles pero el problema hace rato que lo he hecho, ¡no te jode!»

Intercambia burlas con A2 dirigidas a otros compañeros y al profesor. Estirado en la silla, bosteza ruidosamente. En posición apática, se coloca una gorra que le tapa los ojos. Callado, araña la mesa y continúa con burlas sin volver a intervenir en la discusión matemática. Pide al técnico que no le grabe, «ja mí ni me mires, nen!»

Los últimos minutos de la sesión no habla con nadie, apenas gesticula ni se mueve.

\section{Coordenada cronológica}

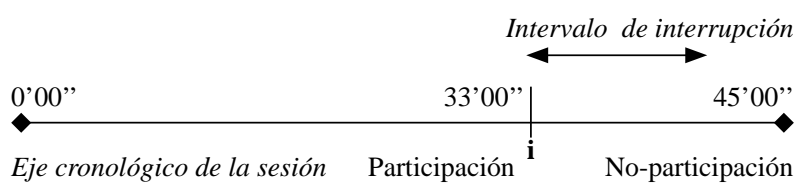

\section{Coordenadas de localización}

Por limitaciones de espacio, no documentamos las coordenadas en su totalidad. Aun así, es posible seguir el estudio del caso. Nos interesa más resaltar el tipo de variables secundarias seleccionadas que comentar sus contenidos específicos.

Contexto del centro: Centro experimental de la reforma educativa; infraestructura física muy degradada; 8 de ratio alumnos-profesor; etc.

Contexto del crédito: La resolución de problemas como centro de interés; problemas organizados en dos bloques alternados sobre proporcionalidad y lógica, trabajo en pequeños grupos seguido de discusión conjunta, etc.

Contexto de la sesión: Primera sesión grabada y cuarta del crédito; martes, de 9'15h a 10'00h; el técnico de vídeo y la observadora son bien recibidos; falta un alumno, el resto se distribuye en los dos grupos de trabajo habituales (ver dibujo); etc.



El profesor reparte fichas con el enunciado del problema:

Queremos cocinar un pastel de manzana. Tenemos la receta con los ingredientes para 3, pero debemos cocinar para 10 invitados. ¿Qué cantidad de cada ingrediente será necesaria?
Ingredientes para 3
1 manzana grande
2 huevos
75 gramos de azúcar
25 gramos de mantequilla
100 gramos de harina
1/2 limón
1 pellizco de sal

Ingredientes para 10 
Perfil del grupo clase: 3 chicas -2 de etnia gitana y 1 de habla castellana- y 3 chicos -2 de etnia gitana y 1 marroquí- ; todos los alumnos hablan el castellano y entienden el catalán aunque no lo usan; pertenecen a familias desestructuradas, etc.

Perfil del alumno (A1): Chico; 17 años; repetidor de $3^{\circ}$ de ESO; sin graduado escolar; expulsado de otro centro por agresión grave y reiterada a infraestructuras; inmigrante marroquí de segunda generación; el profesor lo ve inquieto, agresivo y nervioso; le gustan las matemáticas «porque te hacen pensar hasta volverte loquito». Ayuda a sus hermanos pequeños a hacer los deberes, etc.

Perfil del profesor $(P)$ : Licenciado en biología; 50 años; 25 años de experiencia docente; implicación activa en movimientos de renovación pedagógica, etc.

\section{Construcción de los grafos culturales}

Los grafos culturales organizan información sobre la diversidad de interpretaciones de las normas del aula durante el período de la sesión previo a la interrupción. La construcción de grafos culturales ha de permitir detectar distancias culturales del alumno con otros participantes. Antes de la interrupción, A1 vive al menos tres divergencias al interpretar tres normas del aula: participación del profesor $\left(\mathrm{N}^{1}\right)$, tiempo de resolución de un problema $\left(\mathrm{N}^{2}\right)$ y participación de $\mathrm{A} 1\left(\mathrm{~N}^{3}\right)$. Se elabora y comenta un grafo cultural simple para cada divergencia (Cuadros I, II y III). Por la claridad de sus contenidos, reproducimos los tres grafos sin comentarlos. El grafo cultural compuesto incluye las tres divergencias e introduce respuestas emocionales del alumno al experimentarlas (Cuadro IV). Reproducimos fragmentos de la discusión del grafo compuesto.

\section{Fragmentos de la discusión del grafo cultural compuesto}

El contraste entre la interpretación personal de las normas $\mathrm{N}^{1}, \mathrm{~N}^{2}$ y $\mathrm{N}^{3}$ de $\mathrm{A} 1$ y las del resto de participantes señala múltiples distancias culturales. En relación con $\mathrm{N}^{1}$ (participación de P), existe un importante contraste entre la interpretación de A1, sostenida explícitamente por sus compañeros, y la interpretación simultánea de P. Coexiste una concepción directiva del rol del profesor con una no directiva.

En relación con $\mathrm{N}^{2}$ (tiempo de resolución de un problema), el contraste principal vuelve a producirse entre A1 y P. Mientras A1 y el resto de alumnos esperan una resolución del problema ordenada, unívoca e inequívoca, P opta por conducir una discusión abierta y poco precisa, que sea sugerente pero no concluyente.

En relación con $\mathrm{N}^{3}$ (participación de A1), aparecen dos contrastes significativos cuando A1 toma la palabra para reclamar más atención. P permite que A1 hable a pesar de no integrar sus aportacions, insinuando así la escasa importancia que le atribuye a sus ideas. A2 y A3 aconsejan a A1 que desista de explicar la estrategia de resolución y le recuerdan la conveniencia de que sea $\mathrm{P}$ quien resuelva el problema.
De estas divergencias, surgen unas ciertas distancias culturales. La mayor distancia cultural de A1 es con P, como resultado de las divergencias vividas en la comprensión de las tres normas citadas. La distancia cultural de A1 con A2 y A3 proviene del contraste en torno a $\mathrm{N}^{3}$. La falta de apoyo de A2 y A3 a A1, cuando éste reivindica su autoridad matemática y el derecho a ser tenido en cuenta, crea una distancia que aisla aún más las demandas de A1. Con sus comentarios y gestos, y con la adopción de una actitud pasiva en momentos clave, A2 y A3 asienten de modo acrítico a las observaciones de $\mathrm{P}$ y defienden una actitud paciente y conformista ante el discurso dominante en el aula. A2 y A3 no niegan la validez de los razonamientos de A1, pero parecen considerarlos inoportunos. Por último, no puede decirse que exista una verdadera distancia cultural de A1 con A4 y A5. Las interpretaciones para las normas $\mathrm{N}^{1}$ y $\mathrm{N}^{2}$ son esencialmente convergentes. A4 y A5 muestran señales de coincidencia en las demandas de una mayor directividad y concreción para el proceso de resolución. Respecto a la participación de A1, no expresan opinión alguna. No debe confundirse la relación poco amistosa entre estas alumnas y A1 con juicios emitidos sobre su competencia matemática y sus formas de participación. A4 y A5 escuchan con atención razonamientos de A1 e integran algunas de sus ideas.

\section{Construcción de los grafos sociales}

Para cada grafo cultural simple elaboramos un grafo social simple, de modo que interrelacionamos las categorías cultural y social construidas (Cuadros V, VI y VII). Los grafos sociales organizan información sobre la diversidad de valoraciones dadas a las interpretaciones de las normas que el alumno expresa durante la sesión y antes de la interrupción. La construcción de grafos sociales contribuye a detectar distancias sociales del alumno con otros participantes. Localizamos diversas valoraciones asociadas a las interpretaciones de A1. Cada grafo social simple contiene valoraciones emitidas respecto a una de las normas generadoras de contraste. El grafo social compuesto contiene todas estas valoraciones, la legitimidad de sus emisores y las respuestas emocionales del alumno a dichas valoraciones (Cuadro VIII).

\section{Fragmentos de la discusión del grafo social compuesto}

A lo largo de las divergencias de normas y valoraciones vividas por A1 antes de la interrupción, se generan o refuerzan distancias sociales emergentes entre él y otros participantes que duran toda la sesión sin que se produzcan acercamientos. Esto ocurre en las distancias de A1 con P, A2, A4 y A5. Las dos distancias con P y A2 son similares. Aunque por motivos diferentes (P pretende crear un ambiente de aula concreto y A2 quiere proteger de esfuerzos inútiles a su compañero), ambas distancias vienen marcadas por rechazo a la actitud de A1 y menosprecio por las formas de implicación desmesurada de este alumno en la tarea matemática. P y A2 cuentan con una alta legitimidad en el aula y ello magnifica las distancias. 
Se observa una continua tensión en las formas de contacto de A1 con A4 y A5, falta de cordialidad y confianza. Predominan las burlas y una ausencia de respeto a través de valoraciones negativas expresadas sistemáticamente tras las intervenciones de A1. Esta distancia no parece estar relacionada con la interpretación de las normas de A1, sino con enemistades anteriores. A4 y A5 no reciben especial consideración de A1 ni de P, lo que reduce el impacto de esta distancia social. Por último, la distancia entre A1 y A3 se construye a medida que avanza la sesión, como resultado de sucesivas interacciones de A3 con compañeros que la llevan a modificar la confianza incondicional en A1. Cuando A3 se da cuenta de que la estrategia de A1 es cuestionada, aunque no se han demostrado errores en su enfoque, pasa a considerar poco útiles las ideas de A1 y exagerado su esfuerzo. Esta distancia supone un cambio radical en las relaciones entre A1 y A3, aunque A3 continúa ejerciendo gran influencia en A1.

\section{Construcción del grafo experiencial}

El grafo experiencial es un instrumento, emergente de los grafos culturales y sociales, con una función exploratoria del contexto desde la perspectiva del alumno (Cuadro IX). A partir del contraste de normas y valoraciones en el aula, se elabora el grafo experiencial con el objetivo de controlar las reconstrucciones de las normas que A1 realiza al percibir interpretaciones ajenas y valoraciones no esperadas. Ahora, las respuestas emocionales del grafo cultural compuesto y del social reciben un trato continuo en la configuración de los escenarios emocionales de A1 previos a la interrupción. En este punto, se interrelaciona lo cultural, lo social y lo afectivo.

\section{Fragmentos de la discusión del grafo experiencial}

Hay distancias culturales y sociales que llevan a A1 hasta reconstruir su posición en el aula con un cierto enfado, reafirmando interpretaciones iniciales de dos normas y modificando en parte otra interpretación. Respecto a $\mathrm{N}^{1}, \mathrm{~A} 1$ mantiene su interpretación a pesar de haber comprobado su aislamiento. Aunque P y sus compañeros, excepto A3, le presionan para abandonar su interpretación, la distancia cultural ante el fuerte contraste con Py las valoraciones usadas por éste para ejercer presión sobre A1 no consiguen cambiar su actitud crítica y reivindicativa. La distancia sociocultural asociada a $\mathrm{N}^{1}$ parece potenciar el comportamiento de $\mathrm{A} 1$.

En torno a $\mathrm{N}^{2}$, la distancia cultural, ahora procedente del fuerte contraste con la interpretación de $\mathrm{P}$ y de las valoraciones usadas por éste para ejercer presión sobre el alumno, no modifican la insistencia de A1, quien continúa exigiendo más precisión en el proceso de resolución. Pero esta distancia no actúa como un potenciador del comportamiento de A1, ya que el alumno muestra cansancio e irritación ante el conformismo dominante en el aula. El impacto de esta distancia parece tener un efecto acumulativo sobre la experiencia de la distancia anterior.

Respecto a $\mathrm{N}^{3}$, A1 reconstruye su interpretación inicial al darse cuenta de que la defensa de su estrategia de re- solución requiere aliados en el aula. La distancia cultural y social con P, A2 y A3 y la social con A5 consiguen que A1 deje de pensar que la cuota de participación viene dada por el acierto de sus ideas y se cerciore de que dicha cuota le ha de ser otorgada por los otros y, en especial, por P. Aun así, A1 continúa confiando en la validez de su estrategia. El cambio parcial en su interpretación parece surgir de un proceso creciente de cansancio e irritación iniciado con la vivencia de las dos distancias anteriores. Es inmediatamente después de la reconstrucción de $\mathrm{N}^{3}$ por parte de A1 cuando se produce la interrupción y el alumno deja de intervenir en la tarea matemática.

\section{Elaboración de la ruta explicativa}

Este instrumento reorganiza y sintetiza información de fases anteriores del análisis y explora relaciones emergentes entre distancias culturales, distancias sociales, escenarios emocionales y la posterior interrupción. Reproducimos un esquema de la ruta explicativa (Cuadro $\mathrm{X})$. La columna de la izquierda contiene una secuencia cronológica con las principales distancias culturales detectadas. La columna de la derecha contiene las correspondientes distancias sociales. Una columna intermedia recoge los escenarios emocionales del alumno predominantes en cada momento. La ruta explicativa, al igual que el grafo experiencial, interrelaciona las categorías cultural, social y afectiva, pero ahora con el propósito de vincular las relaciones obtenidas con explicaciones razonables para la interrupción.

\section{Fragmentos de la discusión de la ruta explicativa}

La interrupción puede considerarse como consecuencia del proceso de desánimo creciente y aislamiento de A1. Existen al menos tres motivos desencadenantes que tienen que ver, respectivamente, con las relaciones de A1 y tres miembros del aula: P, A2 y A3. Hay un fuerte contraste de interpretaciones entre $\mathrm{A} 1$ y $\mathrm{P}$ en torno a tres normas esenciales $\left(\mathrm{N}^{1}, \mathrm{~N}^{2}\right.$ y $\left.\mathrm{N}^{3}\right)$. El contraste aparece en medio de dificultades comunicativas ante significados sugeridos por P. El intento de superar las dificultades conduce a nuevas dificultades al negociar normas y valoraciones negativas dadas a A1. La escasa atención de $\mathrm{P}$ a las reivindicaciones de A1 frustra la negociación y provoca que A1 se dirija únicamente a A2 i A3 con la esperanza de conseguir apoyos en su demanda de modificar el comportamiento de P.

El rechazo a $\mathrm{P}$ se exterioriza a través del interés de $\mathrm{A} 1$ por llamar la atención de A2 mientras P se dirige a todos los alumnos. Las relaciones entre A1 y A2 también son problemáticas. Existe una confrontación latente debido a la falta de apoyo que A2 muestra hacia las reivindicaciones de A1. La confrontación manifiesta llega con el establecimiento del significado legitimado de $\mathrm{N}^{3}$. El choque se caracteriza por las dificultades de A1 para comprender la interpretación de A2 y los obstáculos para acercarse a él, ya que A2 evita clarificar sus significados. Estos obstáculos se acumulan a los aparecidos cuando A1 intenta identificar y negociar las interpretaciones de A2 para $\mathrm{N}^{1}$ 
y $\mathrm{N}^{2}$ y discutir las valoraciones negativas que recibe. Sin contar con P ni con A2, A1 opta por refugiarse en A3, con quien mantiene una relación de amistad. Ante la sorpresa de A1, A3 se posiciona con A2 y aparece una repentina confrontación entre A1 y A3. Los esfuerzos de A1 para que no prevalga una interpretación de $\mathrm{N}^{3}$ que contradiga sus intereses chocan con valoraciones negativas de A3 sobre su tozudez y su escaso dominio matemático. La negociación con A3 se inicia creyendo que el ataque inesperado es fruto de una broma pesada, pero la persistencia de A3 cuestiona seriamente este supuesto. A1 se da cuenta de que no puede compartir sus reivindicaciones sobre $\mathrm{N}^{1} \mathrm{y}$ $\mathrm{N}^{2}$ con nadie $\mathrm{y}$, además, no se le permite justificar la estrategia de resolución rechazada por P y sus compañeros.

\section{CONCLUSIONES}

La principal conclusión en relación con la metodología adoptada en la investigación indica que el enfoque metodológico ha sido de gran utilidad para la consecución de los objetivos y la aproximación de respuestas a la cuestión de investigación. Las categorías elaboradas y los instrumentos de análisis son de especial relevancia.

En relación con los instrumentos de análisis construidos especialmente para este estudio, se ha comprobado que son adecuados, cada uno de ellos en diferente medida, en el establecimiento de relaciones entre las perspectivas de lo cultural, lo social y lo afectivo. El carácter complementario de estos instrumentos ha favorecido la visión conjunta e integrada de los diversos temas de interés para cada caso de interrupción y, además, ha aportado dinamismo a los episodios puntuales vividos por el alumno, convirtiéndolos en una secuencia continua con coherencia interna. Por otra parte, la organización de los datos, ya reducidos, por medio de las coordenadas de identificación, de localización de los grafos culturales, los sociales, los experienciales, y las rutas explicativas, ha permitido trabajar simultáneamente los tres temas de interés (interpretaciones de las normas, valoraciones y respuestas emocionales), recuperando cada uno de ellos cuando ha sido necesario y encontrando criterios de clasificación que han facilitado el seguimiento de los casos sin tener que recurrir cada vez a toda la información inicial. Asimismo, los datos de cada caso de interrupción, organizados por medio de los instrumentos mencionados, han sido un factor de triangulación clave al controlar la influencia de los puntos de vista personales de la investigadora durante todo el proceso de simplificación e interpretación.

Respecto a las categorías de análisis, podemos decir que la distinción entre las categorías cultural, social y afectiva, junto con las conexiones establecidas entre ellas al definirlas, ha resultado fundamental para controlar en qué momentos se enfatizaba la dimensión cultural del aula de matemáticas, en qué otros la dimensión social y en qué otros la dimensión emocional de sus participantes, sin que ello haya significado tratar dichas dimensiones por separado. Los datos narrativos recogidos, tanto los relativos a divergencias como a valoraciones o emociones, han sido fácilmente manipulables y los descriptores a los que se han reducido han servido para mostrar significados personales, legitimidades y cambios en los estados de ánimo sin tener que recurrir a complejas contextualizaciones. El análisis de la información de acuerdo con las tres categorías construidas, además de un aproximación microscópica al estudio de cada caso de interrupción, ha supuesto una auténtica aproximación macroscópica a pautas y regularidades aparecidas en los diferentes casos que han servido de punto de partida para reflexionar sobre aspectos generales del aula de matemáticas más allá de alumnos y grupos clase específicos.

Desde el punto de vista del programa de actuación didáctica en el aula, el ambiente de resolución de problemas ha resultado adecuado porque, por una parte, ha facilitado la obtención de información muy rica sobre los temas estudiados y, por otra parte, la mayoría de alumnos ha tenido acceso a las situaciones de aprendizaje propuestas. Los problemas matemáticos escogidos han promovido la participación, al menos inicial, de los alumnos y la proyección frecuente de experiencias personales ha contribuido a mantener la implicación de muchos de ellos. La complejidad de algunos contenidos matemáticos en los problemas y de los procesos cognitivos requeridos para resolverlos parece haber sido, más que un factor generador de bloqueo, un factor que ha llevado a discutir normas vinculadas a la fase de familiarización con el enunciado del problema.

Al analizar a posteriori la adecuación de las decisiones metodológicas conviene hacer una revisión de las limitaciones del estudio. En algunos casos de interrupción podría haber sido conveniente disponer de más datos relativos al alumno. Los dos casos que finalmente no se han conseguido explicar hubieran necesitado muy probablemente de mayor información sobre el alumno tanto desde la perspectiva intrapersonal como interpersonal. $\mathrm{La}$ entrevista individual al alumno, posterior a la sesión de clase en la que se produce la interrupción, hubiera podido contribuir a localizar otras distancias culturales y sociales, así como otros escenarios emocionales, que pueden haber pasado desapercibidos y, a su vez, habría permitido contrastar la interpretación de las distancias y escenarios identificados. Por supuesto, las ventajas metodológicas de introducir más información sobre el alumno hubieran ido acompañadas de los inconvenientes derivados de una mayor complejidad en el conocimiento de éste que no redundaría necesariamente en una interpretación más ajustada.

Por último, y a pesar de que la finalidad principal de este artículo es mostrar resultados relativos a una metodología, introducimos un comentario final acerca de resultados más generales del trabajo. La mayoría de casos de interrupción analizados han sido explicados en base a divergencias en la interpretación de normas sociales del aula de matemáticas. Las normas específicas de la práctica matemática han sido en muy pocas ocasiones generadoras de interrupciones en la participación. De ahí que, en la discusión de los casos, las matemáticas no acostumbren a ser sustantivas. Este resultado permite cuestionar hasta qué punto tiene sentido vincular de manera directa las trayectorias de no-participación en el aula de matemáticas con la comprensión que los alumnos tienen de las prácticas matemáticas específicas. 
Cuadro I

G rafo cultural simple $1\left\{\mathbb{N}^{1}=\right.$ Participación de $P ; I_{X}=$ Interpretación que $X$ hace de $\left.N^{1}\right\}$.

« ¡no te enrolles! di ya cuála está bien que no es tan difícil, venga tío...», «hoy te has tomao un chuti pa desayunar y no lo cuentas... has venío cansao, julai» (cuando P rechaza aclarar dudas)
$\mathrm{I}_{\mathrm{A} 1}=\mathrm{P}$ debe tomar la iniciativa, centrar el tema de discusión e indicar una vía ordenada de aproximación al problema sin confundir intencionadamente a los alumnos.

«muy bien, bueno, yo digo muy bien, pero ya sabéis lo que quiere decir mi muy bien, eso es que estoy escuchando... puede ser muy bien o puede ser muy mal», "¡venga!, ¿qué os ha parecido lo que ha dicho? ¡quiero más ideas! ¡la gandulería pa luego, aquí estáis pa trabajar duro el rato que haga falta, tíos, pa pensar, tíos, que de vez en cuando sienta muy bien... el único que tiene derecho a ser gandul aquí soy yo», "; hay que escoger!» (respuestas habituales a los alumnos)

$\mathrm{I}_{\mathrm{P}}=\mathrm{P}$ debe conducir y enriquecer la discusión, promoviendo la participación de los alumnos y su toma de decisiones

«jvaya mierda con las manzanitas otra vez, venga a liarla y a no decir $n a ! »$ (a A1, en voz baja y haciendo gestos groseros)

$\mathrm{I}_{\mathrm{A} 2}=\mathrm{P}$ no debe confundir a los alumnos con intervenciones ambiguas, desordenadas e incompletas.

"¿oye, profe, esto cómo hay que hacerlo? porfa, di algo, profe, que luego to va a estar muy chungo...", "pero, oye, ¿esto lo hacemos ahora o qué?» (A3 continuamente pide aclaraciones a $\mathrm{P}$ )

$\mathrm{I}_{\mathrm{A} 3}=\mathrm{P}$ debe adoptar una postura directiva e intervenir con mayor frecuencia.

« iy si lo explicas ya, guapito, pa que lo acabemos hoy!» (A4 reacciona con ironía cuando P rechaza aclarar dudas directamente)

$\mathrm{I}_{\mathrm{A} 4}=\mathrm{P}$ debe guardar información importante que impida avanzar en la resolución del problema.

« iya estamos otra vez con el pastel de su abuela, jódete!» (a A4, en voz baja; A5 hace este comentario cuando cree que $\mathrm{P}$ introduce razonamientos no relevantes)

$\mathrm{I}_{\mathrm{A} 5}=\mathrm{P}$ debe alargar los problemas de manera innecesaria y liar a los alumnos sugiriendo ideas que no lleven a ninguna parte. 
Cuadro II

G rafo cultural simple $2\left\{\mathbb{N}^{2}=\right.$ Tiempo de resolución de un problema; $I_{x}=$ Interpretación que $X$ hace de $\left.N^{2}\right\}$.

« ¡oye, tío, así no vamos a acabar ni mañana, esto es pa morirse, tío!», « jesto parece ya como el de las sardinas!», « ¡mira, julai, yo el problema ya hace rato que lo he hecho con el 10 y el molde del colmadero! ¿qué pasa, que no tienes otro preparao pa hoy? ¡te he pillao, sinvergüenza!» (a $\mathrm{P}$, cuando incorpora nuevos elementos del contexto real sugerido por el problema en una fase ya avanzada de la resolución)

$\mathrm{I}_{\mathrm{A} 1}=$ Resolver un problema equivale a encontrar una solución lo más rápidamente posible sin retardar el proceso para encontrar más de una solución.

«vamos a volver a lo que teníamos, hi ha una cosa que no ha quedat gens clara... ¿de verdad que con la sal y el limón hay que ser muy exactos? si no ponemos sal o si no ponemos limón... ¿qué pasa? no sé, yo no lo tengo muy claro», «antes de decidir cuánta harina se necesita, a mí me ha quedado la duda de saber por qué no podemos comprar manzanas pequeñas y grandes, en el mercado y en el súper las manzanas son de diferente tamaño»

$I_{p}=$ Resolver un problema equivale a discutir críticamente posibles soluciones sin prisa y llevar a cabo una constante revisión de las ideas.

«pues claro, eso ya lo sabemos, profe, ¿pero lo redondeamos por arriba o por abajo? ¿cuála está bien?, yo lo veía claro pero yo ya no sé... lo de la manzana grande o pequeña da lo mismo, ¿no? (a P, en voz alta), eso no es importante, te tiene que dar lo mismo» (a A1, en voz baja)

$\mathrm{I}_{\mathrm{A} 3}=$ Resolver un problema equivale a hallar la única solución óptima.

«esto... haces la división y ya está, ¿no, profe?, una división pa cada ingrediente y lo pones escrito debajo de la receta donde hay las rallas y luego lo vamos a comprar al colmadero ese», "¿haces tú (a P) la división o la hago yo?» (reacciona a las preguntas de $\mathrm{P}$ haciendo referencia a operaciones que han de conducir hasta una solución numérica)

$I_{A 5}=$ Resolver un problema equivale a encontrar las operaciones matemáticas que deben ser aplicadas. 
G rafo cultural simple $3\left\{\mathbb{N}^{3}=\right.$ Participación de $A 1 ; I_{X}=$ Interpretación que $X$ hace de $\left.N^{3}\right\}$.

« ‘a mi me es igual, tío (a A2), tú di lo que te dé la gana, yo compro un molde de esos pa 10 personas y voy echando lo que me quepa, lo que sobra lo tiro», «ioye! (a P), ¿te has enterao o no? yo compro un molde pa 10... lo del 9 y el 12 a mí me da igual porque compro un molde pa 10 con el dinero que me queda, eso te juro (a A3) que yo lo he visto y que está bien, lo redondeas cuando compras el molde», « ¿esto es un problema de moldes, que esto yo me lo sé, tío!» (a P; insiste en defender su estrategia), «jtú di lo que quieras, tío» (a P, haciendo un gesto grosero)!

$\mathrm{I}_{\mathrm{A} 1}=$ La resolución del problema requiere la participación de A1

«quiero que todos participéis, que lo hagamos entre todos, vale?» (P da instrucciones de carácter integrador al inicio) «bueno, nos hemos dejao la sal... ¿cómo lo ves (a A4)?» (después de que A1 haya planteado a $\mathrm{P}$ una pregunta relativa a la harina) «lo que dices (a A1) está muy bien, pero a mí me interesa que el colmadero no te tome por loco si les pide 3'3 manzanas, a ver, ¿cuántas manzanas vamos a comprar (a A3)?» «una cosa es decir que ya lo tienes y otra cosa diferente es decir que lo tienes bien hecho...mucha prisa tienes tú hoy (a A1) y mucha charla, esto de salir por la tele no te sienta bien»

$\mathrm{I}_{\mathrm{P}}=\mathrm{A} 1$ es un interlocutor matemático en potencia que excede la cuota de participación, insiste en aspectos irrelevantes y no «oye, tío, déjalo ya, déjate el cerebrín tranquilo, éste te va a marear hasta que toque el timbre y el que sabe el problema es él, ;que lo haga $\quad \mathrm{I}_{\mathrm{A} 2}=\mathrm{La}$ resolución última del problema corresponde él, tío!» (a A1 en voz baja, cuando se da cuenta de su insistencia) a $P$ y cualquier otra aportación no es más que una contribución parcial.

«que sí, que yo lo he oído, tío (A1 le está explicando su estrategia), quedan catorce minutos, tío, ahora lo hace él y lo corregimos...», «déjame, tío, que no me entero de lo que dice el chingao $(\mathrm{P})$ »

$\mathrm{I}_{\mathrm{A} 3}=\mathrm{El}$ acierto de los razonamientos matemáticos de A1 debe ser necesariamente confirmado por $P$. 


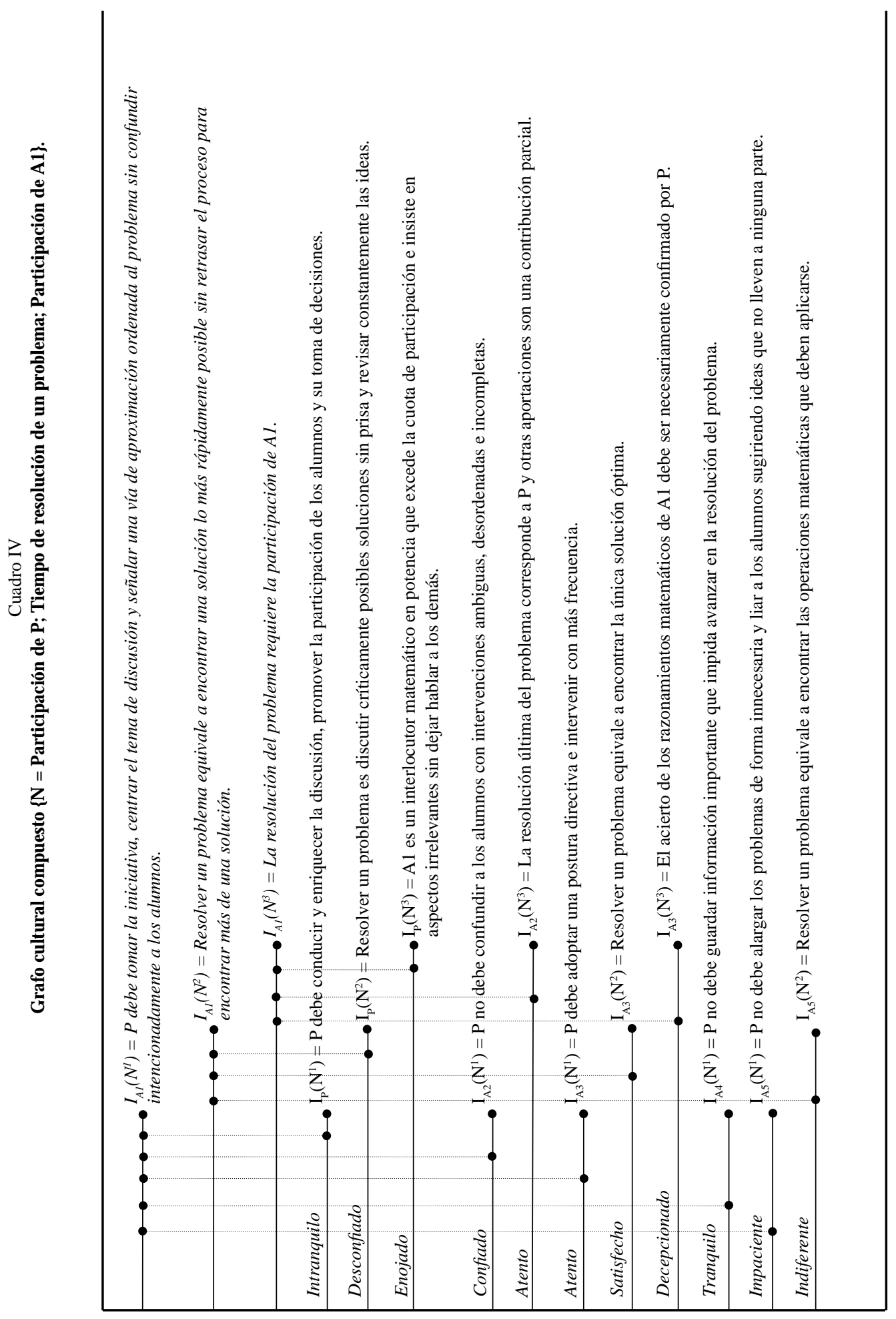


Cuadro V

G rafo social simple $1 \mathbb{N}^{1}=$ Participación de $\mathrm{P} ; \mathrm{V}_{\mathrm{x}}=$ Valoraciones de $\mathrm{X}$ a $\mathrm{I}_{\mathrm{A} 1}$ \}.

$\mathrm{I}_{\mathrm{Al}}=\mathrm{P}$ debe tomar la iniciativa, centrar un tema de discusión y señalar una vía de aproximación al problema de manera ordenada $\mathrm{y}$ sin confundir intencionadamente a los alumnos. «lo que dices está muy bien, muy bien, pero tú quieres que yo hable más de la cuenta... el pastel te lo tienes que hacer tú, bonito, que tienes todo lo que hay que tener, a mí no me necesitas pa na, pensar un poco no perjudica la salud...jeres un pelmazo, tío!» (a A1, cuando reclama más intervención de $\mathrm{P}$ )

$\mathrm{V}_{\mathrm{P}}=(-) \mathrm{A} 1$ quiere que $\mathrm{P}$ participe más porque es inmaduro, gandul y tiene pocas ganas de trabajar.

« ¡déjalo ya, tío, él es así, te explica su vida! ique no te va decir na, tío, aún no te has enterao!... jeres un matao, tío!» $\left(\mathrm{a} \quad \mathrm{V}_{\mathrm{A} 2}=(-) \mathrm{A} 1\right.$ es poco perspicaz porque A1, en voz baja, cuando insiste en pedir más concreción)

« ¡claro, profe! tú tienes que decir cuála está bien... porfa, si no se lo quieres decir a él (A1) pues me lo dices a mí, nen» (cuando A1 pide de nuevo más concreción a P) hace reivindicaciones absurdas e inúti-

les que no le llevan a ninguna parte.

$\mathrm{V}_{\mathrm{A} 3}=(+) \mathrm{A} 1$ se debe escuchar porque hace propuestas que es necesario que alguien explicite.

\section{Cuadro VI}

Grafo social simple $2 \mathbb{N}^{2}=$ Tiempo de resolución de un problema; $V_{x}=$ Valoraciones de $X$ a $I_{A 1}$ \}.

$\mathrm{I}_{\mathrm{A} 1}=$ Resolver un problema equivale a encontrar una solución lo más rápidamente posible. «yo no tengo bastante, yo quiero entender lo que me dice el problema, no tengo bastante con encontrar la solución, ¿me explico?... ir rápido no sirve de nada, con un problema hay que entretenerse, no queda más remedio, tío... piensa lo que vas a decir y a lo mejor no acabas tan rápido» (a A1)

$\mathrm{V}_{\mathrm{P}}=(-) \mathrm{A} 1$ es inmaduro y se precipita al querer saber antes de tiempo la solución del problema.

"ino te lo va a decir hasta que suene el timbre, tío, ahora te va a decir que to está muy bien le digas lo que le digas, mиy bien, muy bien!» (a A1, imitando a P)

«no sé, profe, yo también ya me he liao porque yo pensaba que esto ya estaba bien... que se hacía con el 12... ¿pero cuála está bien, nen? (a $\mathrm{A} 1$ )», "oye (a $\mathrm{A} 1)$ díle que no se enrolle tanto, tío, ;oye, colegui, explícamelo bien, porfa!»,

«a él (a A1) hay que escucharle, pero si eres tú el que le hace una bromita te manda a la mierda... jvaya pelmazo!»

"ino te caduques, tío (a A1), tanta prisa, tanta prisa... que

$\mathrm{V}_{\mathrm{A} 2}=(-) \mathrm{A} 1$ debería ajustarse a las expectativas de $\mathrm{P}$ y no perder el tiempo $\bullet$ haciendo demandas inútiles. el Gerardo te vende un pastel baratito, baratito sin tanto lío, éste $(P)$ ya lo tiene hace rato y esto lo hace pa despistar y liarla, tú di que sí! ja ver por qué tanta prisa, a ti qué más te da mi vida!»
$\mathrm{V}_{\mathrm{A} 2}=(+) \mathrm{A} 1$ se debe tener en cuenta porque expresa en voz alta la confusión de muchos alumnos del aula.

$\mathrm{V}_{\mathrm{A} 4}=(-)$ La insistencia por imponer su opinión muestra su agresividad desmedida.

$\mathrm{V}_{\mathrm{A} 5}=(-) \mathrm{A} 1$ tal vez dice cosas razonables pero no vale la pena que insista tanto en ellas. 
$\mathrm{I}_{\mathrm{A} 1}=$ La resolución del problema requiere la participación de A1. «bien, tú (a A1) ya has dicho lo que querías decir, ahora deja hablar a los demás, que no estás solo», «eso está muy bien, pero yo ya estoy algo mareao con tanto huevo de codorniz y tanto molde, tío... déjalo ya... ", "está muy bien que quieras que te conteste y también que escuches de vez en cuando... si sólo te escucho a ti acabamos pronto, el molde pa 10 y va que chuta...»

$V_{P}=(-)$ La estrategia de A1 es incompleta y sus ansias de participación desmesuradas.

"icállate ya, tío (a A1, en voz baja), si no, no vamos a acabar nunca por tu culpa!... anda, descansa un poquillo y deja que haga el problema él $(\mathrm{P})$ que pa eso le pagan», «relájate, tío, que contigo esto no se acaba» (dando golpes en la espalda de A1 y riendo)

" yo creo que te dice que algo está mal, tío (a A1), lo del molde no te lo dice en la hoja... deja el molde, a ver, esto del molde te lo has inventao, tío.», «no, mira, déjame, que si no, no me entero de na y no le $(\mathrm{P})$ hagas enfadar»

«jeste pastel está tan sobao que no se lo va a comer ni tu madre!», "iya está otra vez el tío este con el molde y el colmado y la madre de su julai...!», "itú (a A1) eres un listillo con los huevos de codorniz!, ;eso ya lo has dicho, tío, cambia el rollo!»

$\mathrm{V}_{\mathrm{A} 5}=(-)$ La estrategia de A1 no es creíble y su participación es anecdótica. 


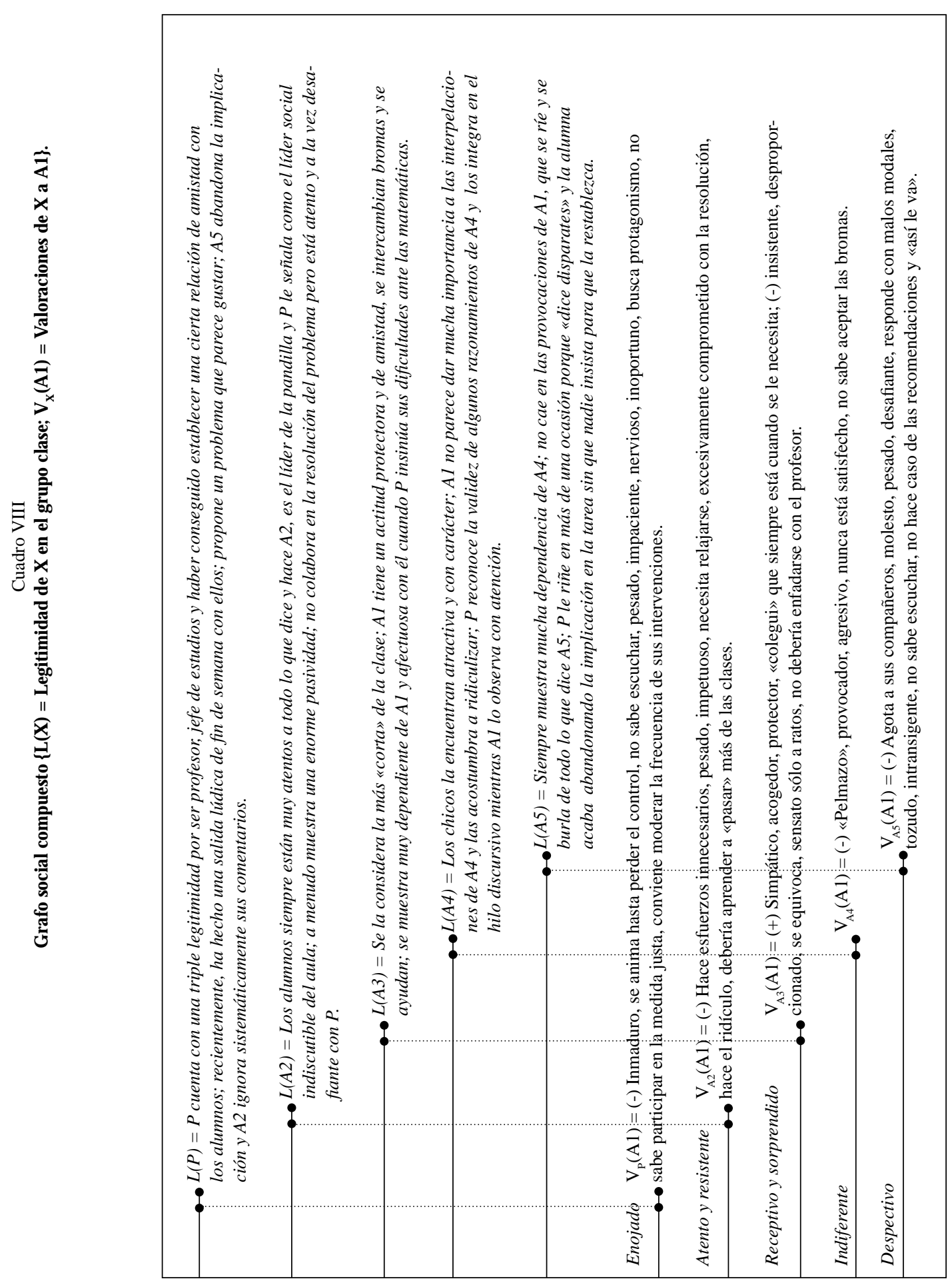




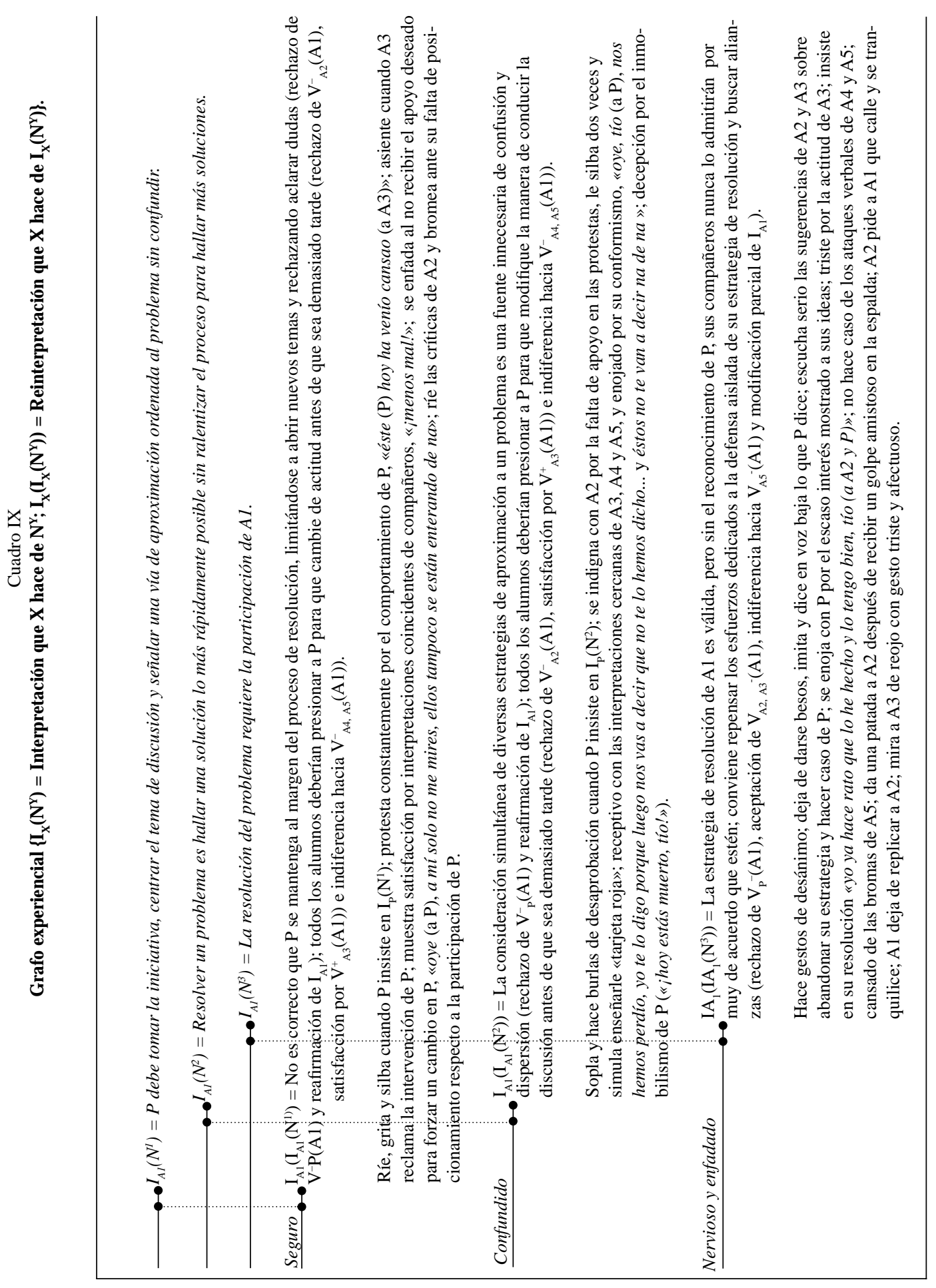


Cuadro X

R esumen de la ruta explicativa.

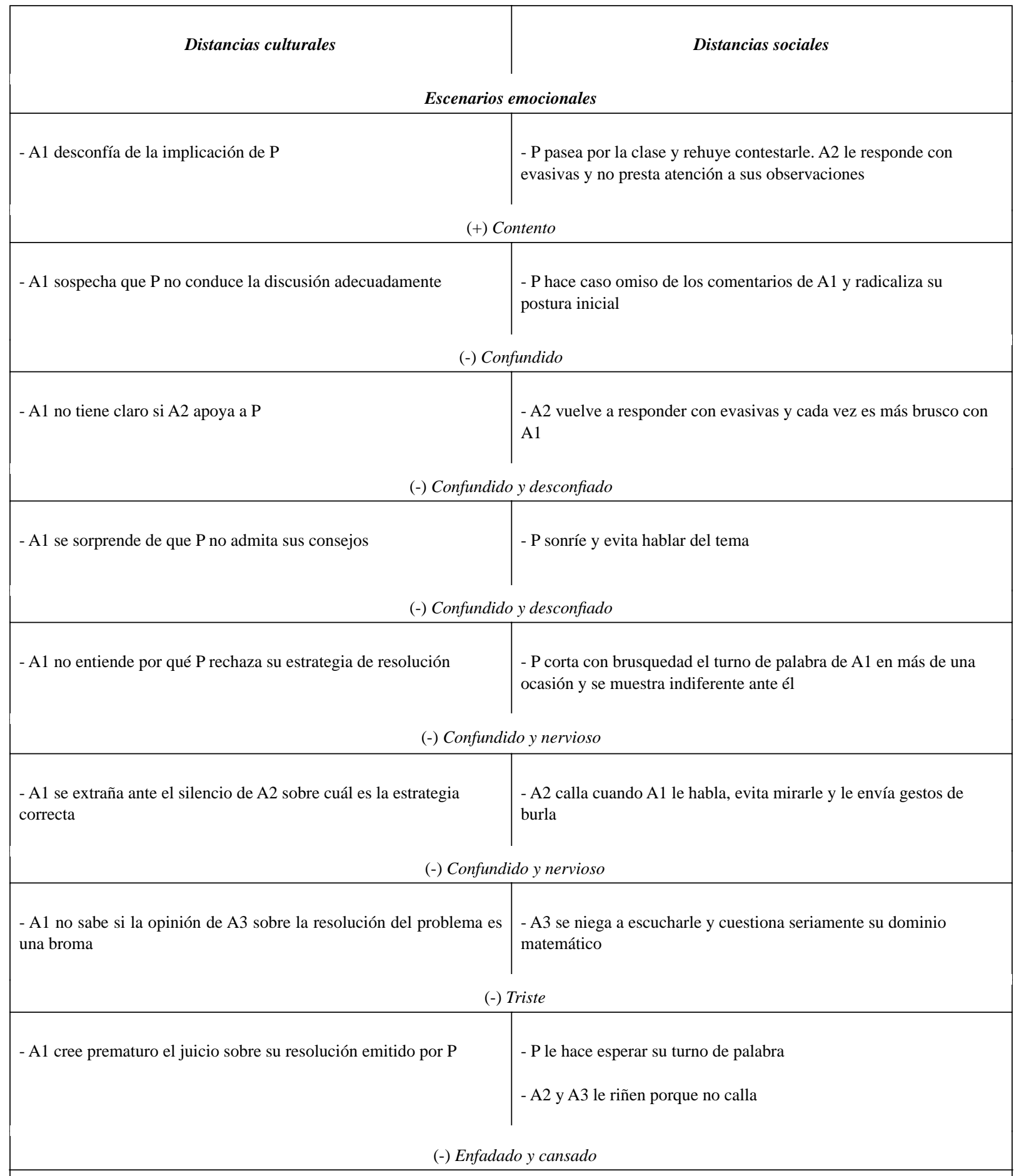

Explicación de la interrupción

La vivencia del contraste en la interpretación de $\mathrm{N}^{1}, \mathrm{~N}^{2}$ y $\mathrm{N}^{3}$ parece indicar que la participación matemática de $\mathrm{A} 1$ a lo largo de la sesión está relacionada con una actitud reivindicativa y, al asumir la pérdida repentina de autoridad por haber introducido una resolución presuntamente errónea, esta actitud ya no tiene sentido y abandona la tarea. 


\section{REFERENCIAS BIBLIOGRÁFICAS}

ABREU, G. (1993). «The relationship between home and school mathematics in a farming community in rural Brazil». Tesis doctoral. University of Cambridge.

ABREU, G. y CLINE, T. (2003). Schooled mathematics and cultural knowledge. Pedagogy, Culture and Society, $11 \mathrm{pp}$. 11-30.

ALRO, H. y SKOVSMOSE, O. (2002). Dialogue and learning in mathematics education. Dordrecht: Kluwer.

BOALER, J. y GREENO, J. (2000). Identity, agency and knowing in mathematics worlds, en Boaler, J. (ed.). Multiple perspectives on mathematics teaching and learning, pp. 171-200. Westport: Ablex.

CLARKE, D.J. (1998). Studying the classroom negotiation of meaning: Complementary accounts methodology, en Teppo, A. (ed.). Qualitative research methods in mathematics education. Reston, Virginia: NCTM, Monograph 9.

COHEN, L. MANION, L. y MORRISON, K. (1997). Research methods in education. Londres: Routledge.

COBB, P. y YACKEL, E. (1998). A constructivist perspective on the culture of the mathematics classroom, en Seeger, F. et. al. (eds.). The culture of the mathematics classroom, pp. 158-190. Cambridge: Cambridge University Press.

EVANS, J. (2000) Adults' mathematical thinking and emotions: A study of numerate practices. Londres: Routledge.

LERMAN, S. (2000). The social turn in the mathematics education research, en Boaler, J. (ed.). Multiple perspectives on mathematics teaching and learning, pp. 19-44. Westport: Ablex.

LERMAN, S. (2001). Cultural, discursive psychology: A sociocultural approach to studying the teaching and learning of mathematics. Educational Studies in Mathematics, 46(1/3), pp. 87-113.
MORGAN, C. (2000). Discourses of assessment-discourses of mathematics, en Matos, J.P. y Santos, M. (eds.). Proceedings of the $2^{\text {nd }}$ International Mathematics Education and Society Conference, pp. 58-76. Montechoro (Portugal).

PLANAS, N. (1999). «Ambient de resolució de problemes en una classe multiètnica: identificació de norma social, norma sociomatemàtica i norma matemàtica». Tesis de maestría. Universitat Autònoma de Barcelona.

PLANAS, N. (2001). «Obstacles en l'aprenentatge matemàtic: la diversitat d'interpretacions de la norma». Tesis doctoral. Universitat Autònoma de Barcelona. www.tdcat.cbuc.es/ TDCat-1116101-145701/index.html

PLANAS, N. (2002). Obstáculos en el aprendizaje matemático generadores de interrupciones en la participación. Educación Matemática, 14(1), pp. 5-25.

PLANAS, N. y CIVIL, M. (2002). Understanding interruption in the mathematics classroom: Implications for equity. Mathematics Education Research Journal, 14(3), pp. 169189.

WENGER, E. (1998). Communities of practice: Learning, meaning and identity. Cambridge: Cambridge University Press.

WOOD, T. (1994). Patterns of interaction and the culture of mathematics classrooms, en Lerman, S. (ed.). Cultural perspectives on the mathematics classroom, pp. 149-168. Dordrecht: Kluwer.

YACKEL, E. y COBB, P. (1996). Sociomathematical norms, argumentation and autonomy in mathematics. Journal for Research in Mathematics Education, 27(4), pp. 458-477.

ZEVENBERGEN, R. (2002). Streaming in school mathematics: A Bourdieuian analysis, en www.congress-consult.com/mes3/ full_program.html\#All Documents. 